\title{
Reply to Comment on Margins in Breast-Conserving Surgery After Neoadjuvant Therapy
}

\author{
Tari A. King, $\mathrm{MD}^{1,2}$ \\ ${ }^{1}$ Associate Chair for Multidisciplinary Oncology, Division of Breast Surgery, Department of Surgery, Brigham and \\ Women's Hospital, Dana-Farber/Brigham and Women's Cancer Center, Boston, MA; ${ }^{2}$ Anne E. Dyson Professor of \\ Surgery in the Field of Women's Cancers, Harvard Medical School, Boston, MA
}

To the Editor:

We are grateful to Drs. Wenxiang Zhang, Xiangyu Wang, Yi Fang, and Jing Wang for carefully reading our manuscript "Margins in Breast-Conserving Surgery after Neoadjuvant Therapy." They have identified an error in the following statement from the Results section: "Local recurrence occurred in 3 of $103(2.9 \%)$ patients with 1.1- to 2-mm margins, 11 of $174(6.3 \%)$ patients with $>2$-mm margins, and 1 of $105(1.0 \%)$ patients with a breast pCR."
Because our primary analysis compared patients with $\leq 2$-mm margins, $>2$-mm margins, and breast $\mathrm{pCR}$, this sentence should have stated: "Local recurrence occurred in 3 of 103 (2.9\%) patients with $\leq 2$-mm margins, 11 of 174 (6.3\%) patients with $>2$-mm margins, and 1 of $105(1.0 \%)$ patients with a breast pCR." As the readers describe, while unfortunate, this error does not change the results of our primary analysis nor the conclusions of the manuscript.

(C) Society of Surgical Oncology 2020

First Received: 21 August 2020

Accepted: 21 August 2020;

Published Online: 30 August 2020

T. A. King, MD

e-mail: tking7@bwh.harvard.edu 